\title{
Intraoperative transvaginal sonography: a novel approach for localization of deeper myomas during laparoscopic myomectomy
}

\author{
P.G Paul ${ }^{1}$ - Dimple K. Ahluwalia ${ }^{1}$ Dhivya Narasimhan ${ }^{1}$ - Gaurav Chopade ${ }^{1}$. \\ Saurabh Patil ${ }^{1}$ - Varsha Rengaraj ${ }^{1}$ • Tanuka Das ${ }^{1}$
}

Received: 9 September 2014 / Accepted: 2 September 2015 /Published online: 11 September 2015

(C) Springer-Verlag Berlin Heidelberg 2015

\begin{abstract}
The aim of this study is to assess the use of intraoperative transvaginal ultrasonography (TVS) to locate deep myomas that were not identified on laparoscopic view. The design of this study is a prospective observational study. This study was conducted in private Advanced Endoscopy and Infertility Treatment Centre, Kerala, India. The study comprised of 84 patients who underwent laparoscopic myomectomy from January 2011 to December 2013 in whom intraoperative TVS was used as an intervention. The number of additional deeper myomas removed was calculated, and recurrence at 1 year was calculated. The total number of myomas enucleated was 390 , and the additional myomas enucleated after intraoperative TVS were 94. The recurrence of myomas at 1 -year follow-up was $7.1 \%$. Intraoperative TVS was helpful to the surgeon for identifying deeper myomas making the surgery more effective.
\end{abstract}

Keywords Myomas · Intraoperative TVS · Laparoscopic myomectomy

\section{Introduction}

Laparoscopic myomectomy was described for the first time in the late 1970s for subserous myomas [1]. In the early 1990s,

P.G Paul

drpaulpg@gmail.com

1 Centre for Advanced Endoscopy and Infertility, Paul's Hospital, Vattekkattu Road, Kaloor, Kochi, Kerala 682 017, India the technique was developed to include removal of intramural myomas [2]. As endoscopic surgeons gained experience, they started performing laparoscopic myomectomy for multiple and larger myomas, irrespective of size, number, or location of myoma [3].

One of the difficulties of laparoscopic myomectomy is locating the deeper and smaller myomas especially those closer to the endometrium (type 3) according to the International Federation of Gynecology and Obstetrics (FIGO) classification [4]. Laparoscopic myomectomy carries increased risk of residual myomas because unlike laparotomy, the uterus cannot be palpated to locate very small myomas. Postoperative recurrence may be either due to enlarged residual myomas or newly formed myomas. There are studies that show increased risk of recurrence $(16.7-51.4 \%)$ after 5 years of laparoscopic myomectomy $[5,6]$. This increases the chance of reoperation and decreases the chances of symptom relief after the surgery. Several studies have shown that large myomas are associated with significant reduction in pregnancy rate after IVF [7, 8]. Khalaf et al. showed that smaller myomas $(\leq 5 \mathrm{~cm})$ not encroaching endometrial cavity were found to significantly reduce ongoing pregnancy rate at each cycle of IVF by $40 \%$; similarly, Stovall et al. concluded that implantation and pregnancy rates were one half that of matched controls $[9,10]$.

Technical problems in identifying deeper myomas lead to misplaced incision causing more blood loss, myometrial integrity, and increased operating time. Good preoperative myoma mapping is helpful, but it is difficult to locate deeper and smaller intramural myomas intraoperatively [11]. Intraoperative location of myomas with laparoscopic contact ultrasound probes can be done [12]. But it is costly and not available as standard ultrasound probes. To overcome this, we attempted to use intraoperative transvaginal ultrasonography (TVS) with 
a simultaneous laparoscopic view to locate deep-seated and smaller myomas and to enucleate additional residual myomas.

The primary aim of the study was to assess the effectiveness of intraoperative transvaginal ultrasonography to locate deeper and smaller myomas, which were not identified on laparoscopic view.

\section{Material and methods}

This was a prospective observational study of women who underwent laparoscopic myomectomy for uterine leiomyomas from January 2011 to December 2013 when intraoperative TVS was done to identify the additional myomas.

For all patients, preoperative TVS and transabdominal scans were performed, and inclusion criteria were:

1) Patients with four or more myomas.

2) Patients with type 3 myoma, irrespective of the number of myomas (myoma that contacts the endometrium and is $100 \%$ intramural) according to the FIGO classification [4].
Exclusion criteria were:

1) Patients with other coexisting diseases like endometriosis and severe pelvic adhesion.

2) Patients with submucous myomas type 0,1 , and 2 according to FIGO classification.

3) Postmenopausal women.

Laparoscopic myomectomy was not limited by factors such as location (anterior/posterior wall) and depth (subserosal/intramural). Data was collected on demographic characteristics, and the chief indication and symptoms were analyzed. The study was approved by the ethical committee of Paul's Hospital for the intervention.

Preoperatively GnRH agonists were not used before surgery because we found that degeneration of the myoma made the surgical dissection difficult. Postoperative patients were followed up with TVS by the first author for recurrence of myomas at 1 year, and any myoma of more than $2 \mathrm{~cm}$ was considered as recurrence (Fig. 1).
Fig. 1 Flow chart of patients from selection to 1 year after myomectomy

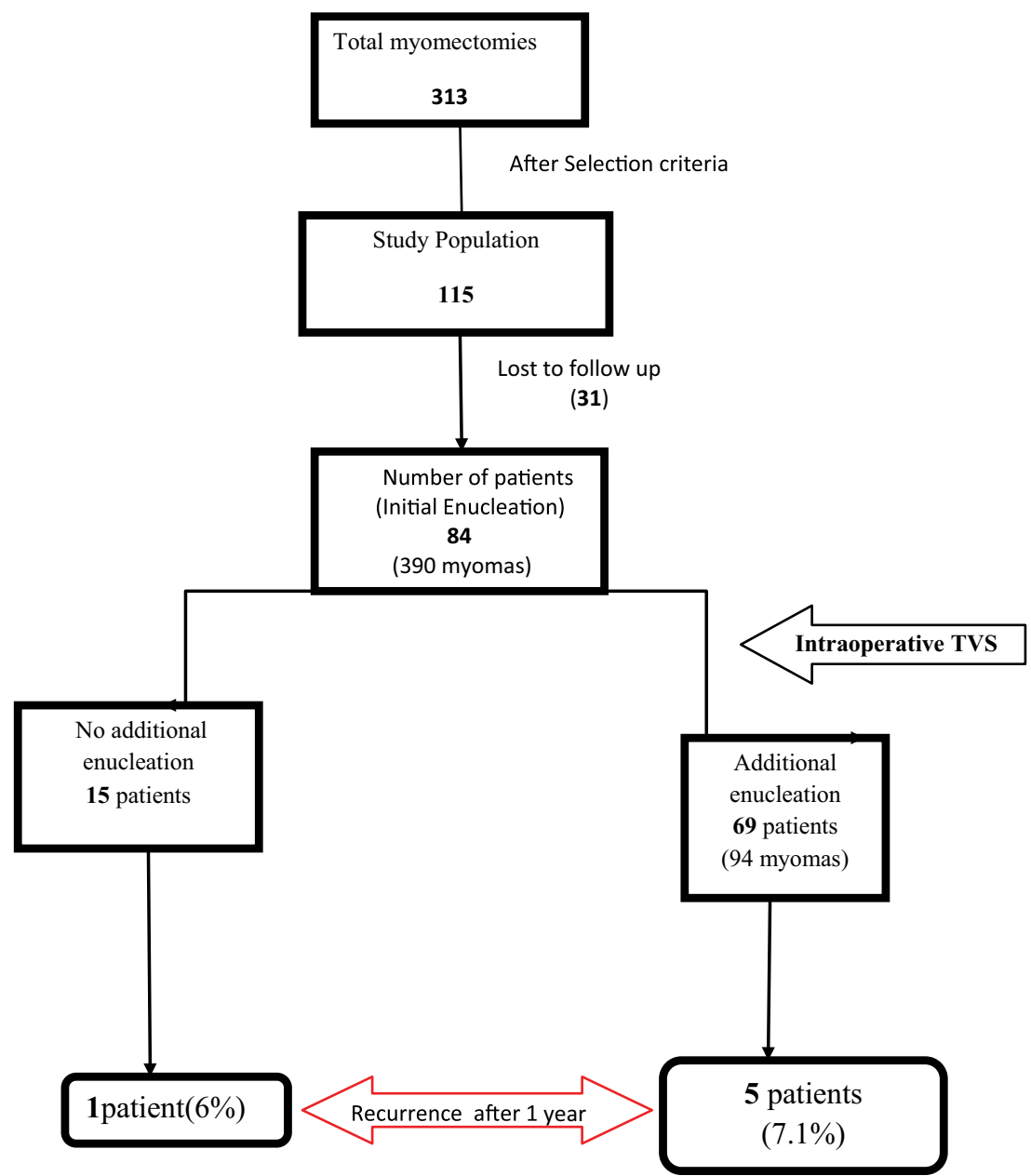


Fig. 2 Deep intramural myoma mapped preoperatively

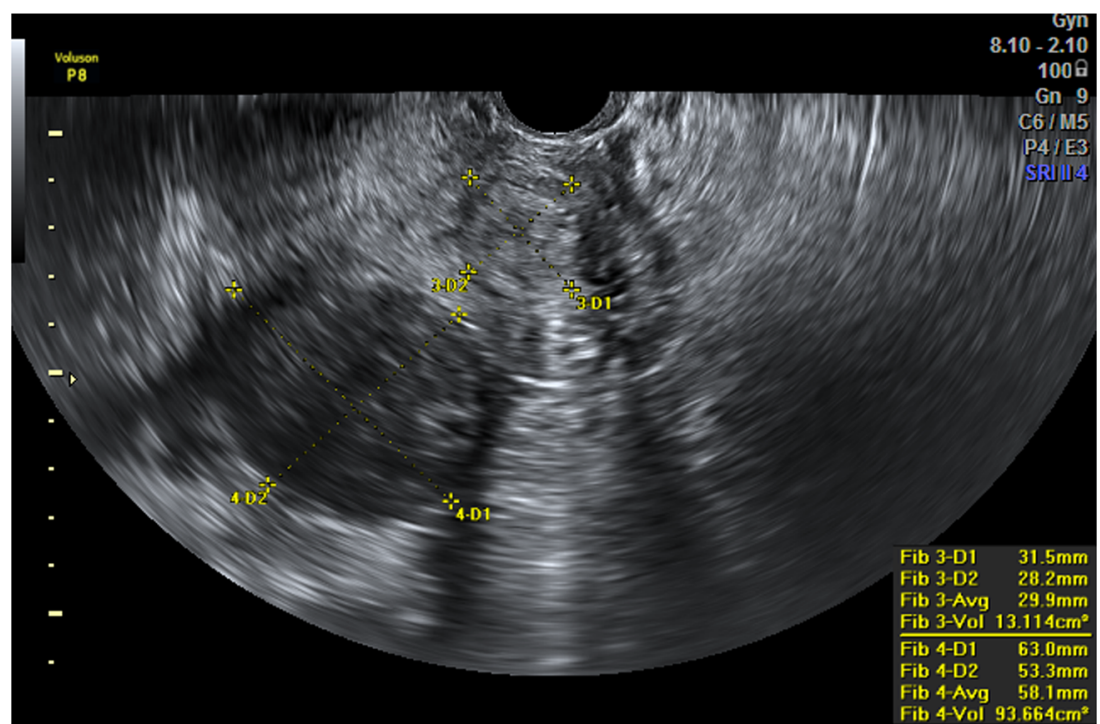

\section{Surgical technique}

All procedures were performed by the first author using similar technique under general anesthesia, as described in our previous publications $[13,14]$. Hysteroscopy was performed to look for distortion of the endometrial cavity and to exclude any undiagnosed submucous myoma on ultrasonography. Entry into the abdominal cavity through an umbilical incision or a higher one in the case of larger uteri was accomplished using a $10-\mathrm{mm}$ trocar. In patients with a previous history of an open surgery or in cases where intra-abdominal adhesions were suspected, entry under direct vision using a Ternamian Endotip (Karl Storz, Tuttlingen, Germany) was performed. Two ancillary 5-mm trocars lateral to the right and left epigastric vessels and a median supra-pubic trocar were inserted. Vasopressin $20 \mathrm{IU}$ diluted in $60 \mathrm{ml}$ of saline was infused into the myometrium of the uterus to reduce the bleeding. A transverse or vertical incision was made over the myoma that was preoperatively mapped (Fig. 2) with Harmonic Ace (Ethicon Endosurgery, Cincinnati, Ohio).The myoma was kept under traction using myoma spiral and dissected with Harmonic Ace. The myomectomy site was sutured intracorporeally with polyglactin 910 (Vicryl, Ethicon, India) or 1-0 braided Lactomer (Polysorb, Tyco Healthcare) in single or double layers depending upon the depth of muscle defect. The myomas were removed with electromechanical morcellator (Karl Storz, Gynaecare, Tuttlingen, Germany). After enucleating all visible myomas, the intraoperative TVS was done, and additional myomas were identified.

Intraoperative TVS was done by either a second or third author and confirmed by an operating surgeon (first author). All preoperative TVS were done by the first author. We used
Fig. 3 Deep intramural myoma identified by intraoperative sonography

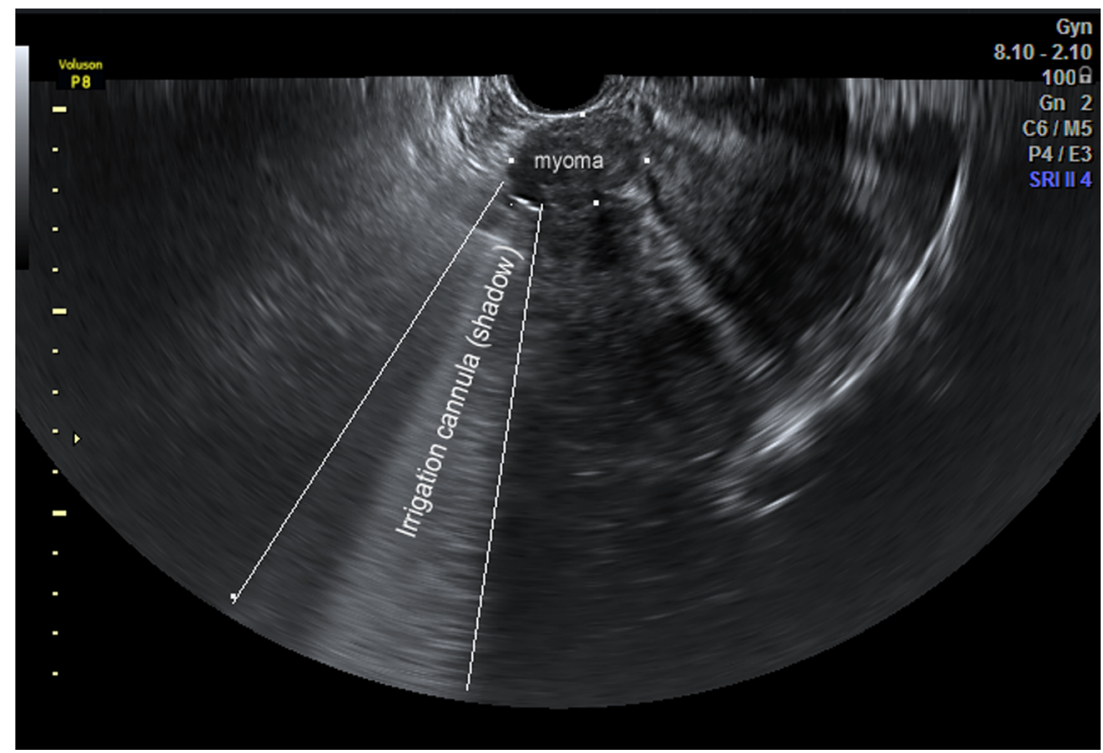


ultrasonic device Voluson E (GE Healthcare, Beethoven Street, 239 D m- 42,655, Solingen, Germany) with TVS probe RIC5-9W-RS/Gyn and detection frequency set at 8-14 MHz. The vaginal probe was covered with a sterile cover, and the whole uterus was scanned to identify any residual myoma. The laparoscopic surgeon (first author) filled the pouch of Douglas with normal saline to make an acoustic window for better ultrasonic visualization. The myoma identified on transvaginal ultrasound was located laparoscopically with a suction irrigation cannula by trial and error. The irrigating fluid from suction irrigation cannula seen as a comet shape on transvaginal ultrasound helps in the localization of the myoma (Fig. 3). The myoma thus identified was enucleated laparoscopically as described before (Fig. 4).

\section{Results}

The total number of myomectomies performed from January 2011 to December 2013 was 313. One hundred fifteen patients met inclusion criteria, but 31 patients were lost to follow-up; of these, 84 patients who met the inclusion criteria were included in the study. The mean age of our patients was $33.40 \pm 5.13$ and mean BMI was 32. The proportion of parity was $52 \%$. The primary preoperative indications were abdominal pain in 35 patients $(41.7 \%$ ) followed by primary infertility in 22 patients $(13.1 \%)$ and menorrhagia in 14 patients $(16.7 \%)$ and as shown in Table 1.

The size of the myomas enucleated is shown in Table 2. The average number of myomas enucleated per patient was 4.64. Sixty-three patients had four or more myomas. Twentyone patients had less than four myomas, but these were all type 3.

The total number of myomas removed was 390 . The mean size of the myomas removed was $3.28 \mathrm{~cm}$.

The average blood loss in milliliter was $163.86 \pm 18.92$ in patients with intraoperative TVS, whereas $148.18 \pm 21.44$

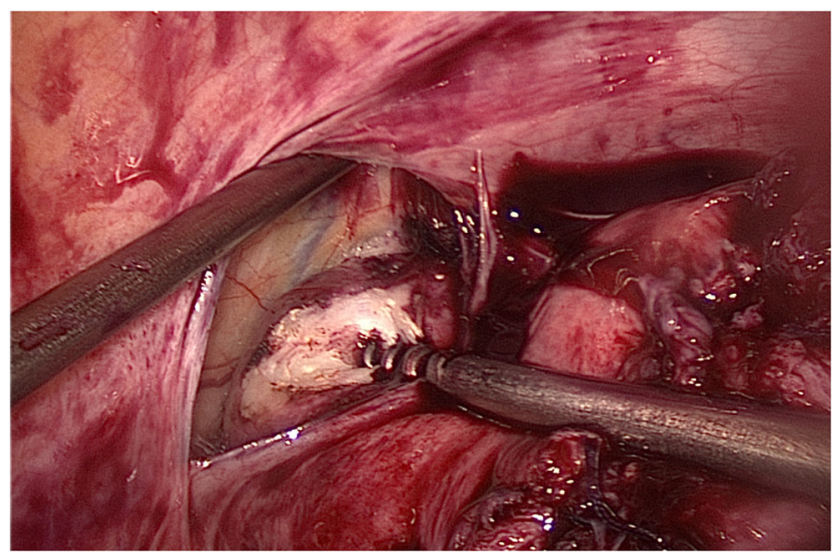

Fig. 4 Laparoscopic enucleation of deep intramural myoma
Table 1 Demographic characteristics/chief complaints

Demographic characteristics

\begin{tabular}{ll}
\hline Age (years) & $33.40 \pm 5.13$ \\
BMI (mean) & 32 \\
Chief complaints & No. of patients \\
Abdominal pain & $35(41.7 \%)$ \\
Primary infertility & $22(26.2 \%)$ \\
Menorrhagia & $14(16.6 \%)$ \\
Secondary infertility & $4(4.8 \%)$ \\
Dysmenorrhea & $5(5.9 \%)$ \\
Dysmenorrhea and menorrhagia & $2(2.4 \%)$ \\
Irregular bleeding & $2(2.4 \%)$
\end{tabular}

without intraoperative TVS. The mean drop in hemoglobin concentration after surgery was $1 \mathrm{~g} \%$. The total time duration (induction to closure) was $134 \pm 27.25 \mathrm{~min}$ in cases where intraoperative TVS was performed and $110 \pm 21.02 \mathrm{~min}$ in cases without intraoperative TVS.

The additional myomas removed after performing intraoperative TVS were 94 . These were visible during preoperative TVS also. Size (range) of the additional myomas removed was $2.5(1.5-3.5 \mathrm{~cm})$. Average number of additional myomas enucleated per patient was 1.1. Out of 94 additional myomas removed, 86 were FIGO type III and 8 were type IV. Recurrence of myoma at 1-year follow-up on ultrasound examination was $7.1 \%$.

\section{Discussion}

In patients with multiple myomas, an experienced laparoscopic surgeon can remove all visible myomas but identification of deeper and smaller myomas are difficult due to lack of tactile perception. The better way to overcome this limitation is the

Table 2 Total number of myomas

\begin{tabular}{lc}
\hline Size $(\mathrm{cm})$ & Number of myomas \\
\hline$<5$ & $324(83.08 \%)$ \\
$5-6$ & $20(5.13 \%)$ \\
$6-7$ & $20(5.13 \%)$ \\
$7-8$ & $9(2.31 \%)$ \\
8 & $9(2.31 \%)$ \\
9 & $3(0.77 \%)$ \\
10 & $1(0.26 \%)$ \\
12 & $1(0.26 \%)$ \\
14 & $3(0.77 \%)$ \\
Mean size $=3.28 \mathrm{~cm}$ & Total -390 myomas
\end{tabular}


Table 3 Intraoperative findings

\begin{tabular}{llr}
\hline Intraoperative findings & With intraoperative TVS & Without intraoperative TVS \\
\hline Total time duration (min) & $134.63 \pm 27.25$ & $110 \pm 21.02$ \\
Total blood loss (ml) & $163.86 \pm 18.92$ & $148.18 \pm 21.44$ \\
Number of additional myomas removed & 1.1 & \\
\hline
\end{tabular}

use of intraoperative ultrasonography. The aim of the study was to assess the effectiveness of intraoperative TVS to locate deeper and smaller myomas that were not identified on laparoscopic view. This is in contrast to a similar study where an intraoperative contact probe was used. However, this method is costly and not easily available [12]. To our knowledge, this is the first study where TVS is used intraoperatively to localize deep/hidden myomas.

In our study, mean size of myomas removed was $3.28 \mathrm{~cm}$, which is not considered significant by Pritts et al. in his review article. Most of our patients were having a long duration of infertility and planning to go for IVF and that was the reason for removing smaller myomas. The size of the myomas removed is similar to other studies [9, 10, 15]. Additional myomas that were not visible laparoscopically were detected by intraoperative TVS in 69 of 84 patients. The mean size of the additional enucleated myomas was 2.5 (1.5 to $3.5 \mathrm{~cm})$. In a similar study where intraoperative contact ultrasound probe was used, 25 additional myomas with a median diameter of $1.2 \mathrm{~cm}$ were enucleated in a group of 42 patients which is comparable to our study [12].

In our study, the operative time was more when intraoperative TVS was done due to the time taken for sonography and the surgical time for enucleating additional myomas. The blood loss was also more due to increased duration of the surgery. (Table 3) But blood loss was not clinically significant, and duration can be minimized with experience in intraoperative TVS.

Causes of postoperative recurrence are considered to be either enlargement of residual myomas or formation of new ones. Postoperative residual myomas greatly affect the recurrence rate. Some studies have reported that laparoscopic myomectomy is associated with a higher recurrence rate as compared with laparotomy recurrence rate $[16,17]$. Doriot et al. and Hiroto Shimanuki et al. defined recurrence of a myoma $>2 \mathrm{~cm}$ with transvaginal ultrasonography to be significant in their study after laparoscopic myomectomy [12]. In a study by Rossetti et al. which compares recurrence of myomas after 6 months in the abdominal and the laparoscopic group, there was a recurrence of $27 \%$ in the laparoscopic group as compared to $23 \%$ in the abdominal group [16]. In our study, recurrence at 1year follow-up was $7.1 \%$ that is lower than the above studies. In our study, recurrence was due to the formation of new ones as we had removed visible myomas.
The intraoperative ultrasound allowed precise localization of the myoma and determination of the best hysterotomy incision. A suboptimal incision would have caused greater trauma to the normal myometrium, as well as increased operating time for laparoscopic reconstruction of the uterus following the myomectomy. The other option would have been converting to an open procedure to enable palpation of the location of the known myomas and making an appropriate incision. Laparoscopic myomectomy is a well-accepted surgical approach for selected patients [16]. The intraoperative TVS allowed the surgeon to complete the myomectomy laparoscopically without tactile information.

Transvaginal sonography is a widely available imaging modality that every gynecologist is well versed with although there can be an interobserver variation. Its novel use intraoperatively to localize deep-seated myomas enables the surgeon to complete the myomectomy laparoscopically, despite the absence of tactile sensation. Limitation of the study was a lack of randomization and small sample size due to lost to followup (Fig. 1) and short follow-up time (1 year).

\section{Conclusion}

Intraoperative TVS is helpful to the surgeon for identifying deeper and smaller myomas, thus making the surgery more effective. Hence, intraoperative use of transvaginal sonography for patients with multiple and deep-seated myomas is advantageous.

Conflict of interest The authors declare that they have no competing interests. The authors alone handle the content and writing of the paper.

Informed consent All procedures followed were approved by the ethical standards of the responsible committee on human experimentation (institutional and national) and in accordance with the Helsinki Declaration of 1975, as revised in 2000(5). Informed consent was obtained from all patients for being included in the study.

Authors'contributions Dr. P.G Paul was the operating surgeon responsible for the planning and conduct of research work. Dr. Dimple K. Ahluwalia and Dr. Dhivya Narasimhan were the assistant surgeons assigned in performing ultrasound during surgeries and conducting studies. Dr. Gaurav Chopade performed statistical analysis and also helped in conducting studies. Dr. Saurabh Patil took charge of the reporting and review of literature. Dr. Varsha Rengaraj and Dr. Tanuka Das were responsible for the preparation of the manuscript. 


\section{References}

1. Semm K, Mettler L, et al (1980) Technical progress in pelvic surgery via operative laparoscopy. Am J Obstet Gynecol 138:121-127

2. Daniell JF, Gurley LD, et al (1991) Laparoscopic treatment of clinically significant symptomatic uterine myomas. J Gynecol. Surg 7: 37-39

3. Sinha R, Hegde A, Mahajan C, Dubey N, Sundaram M (2008) Laparoscopic myomectomy: do size, number, and location of the myomas form limiting factors for laparoscopic myomectomy? J Minim Invasive Gynecol 15:292-300

4. Munro MG et al (2011) FIGO Working Group on Menstrual Disorders: FIGO classification system (PALM-COEIN) for causes of abnormal uterine bleeding in nongravid women of reproductive age. Int J of Gynecol \& Obstet. 113:3-13. doi:10.1016/j.ijgo.2010. 11.011

5. Doridot V, Dubisson J, Chapron C, et al (2001) Recurrence of leiomyomata after laparoscopic myomectomy. J Am Assoc Gynecol Laparosc 8:495-500

6. Nehzat FR, Roemisch M, Nezhat CH, et al (2001) Recurrence rate after laparoscopic myomectomy. J Am Assoc Gynecol Laparosc 8: 495-500

7. Benecke C, Kruger TF, Siebert TI, Van der Merwe JP, Steyn DW (2005) Effect of fibroids on fertility in patients undergoing assisted reproduction. A structured literature review. Gynecol Obstet Investig 59:225-230

8. Pritts EA, Parker WH, Olive DL (2009) Fibroids and infertility: an updated systematic review of the evidence. Fertil Steril 91:12151223
9. Khalaf Y, Ross C, El-Toukhy TH, Seed RP, Braude P, et al (2006) The effect of small intramural fibroids on the cumulative outcome of assisted conception. Hum Reprod 21:2640-2044

10. Stovall DW, Parrish SB, Van Voorhis BJ, et al (1998) Uterine leiomyomas reduce the efficacy of assisted reproduction cycles: results of a matched follow-up. Study; Hum Reprod vol.13(no.1): 192-197

11. Dubuisson JB, Fauconnier A (2007) Laparoscopic myomectomy. In: Atlas of operative laparoscopy and hysteroscopy. 3rd edn, Informa healthcare, U.K, p. 235

12. Shimanuki H et al (2006) Effectiveness of intraoperative ultrasound in reducing recurrent fibroids during laparoscopic myomectomy. J Reprod Med 51:683-688

13. Paul PG, Koshy A, Thomas T, et al (2006) Pregnancy outcomes following laparoscopic myomectomy and single layer myometrial closure. Hum Reprod 21:3278-3281

14. Paul PG, Koshy A, Thomas T, et al (2006) Laparoscopic myomectomy: feasibility and safety - a retrospective study of 762 cases. Gynecol Surg 3:97-102

15. Liselotte M, Thoralf Schollmeyer E, et al (2005) Update on laparoscopic myomectomy. Gynecol Surg 2:173-177

16. Rosette A, Sizzi O, et al (2001) Long-term results of laparoscopic myomectomy: recurrence rate in comparison with abdominal myomectomy. Hum Reprod 16:770-774

17. Miller CE et al (2000) Myomectomy. Comparison of open and laparoscopic techniques. Obstet Gynynecol Clin North Am 27: $407-420$ 